\title{
PRONGS: Powerful Radio Objects Nested in Galaxies with Star Formation
}

\author{
Minnie Y. Mao ${ }^{1,2,3}$, Ray P. Norris ${ }^{2}$, Rob Sharp ${ }^{3}$, and Jim E. J. Lovell ${ }^{1}$ \\ ${ }^{1}$ School of Maths \& Physics, University of Tasmania, Private Bag 37, Hobart, 7001, Australia \\ Email: mymao@utas.edu.au \\ ${ }^{2}$ CSIRO Australia Telescope National Facility, PO Box 76, Epping, NSW, 1710, Australia \\ ${ }^{3}$ Anglo-Australian Observatory, PO Box 296, Epping, NSW, 1710, Australia
}

\begin{abstract}
We have identified a new class of object that we term PRONGS (powerful radio objects nested in galaxies with star formation). These are powerful radio sources whose optical properties are that of spiral/star-forming galaxies, unlike classic powerful radio sources which are typically hosted by elliptical galaxies in the local Universe. Here we present a first look at these enigmatic sources.
\end{abstract}

Keywords. galaxies: general, galaxies: active, galaxies: starburst, radio continuum: galaxies

Giant double-lobed radio sources are almost always hosted by elliptical galaxies (e.g., Matthews, Morgan, \& Schmidt 1964). We have discovered in ATLAS, the Australia Telescope Large Area Survey (Norris et al. 2006; Middelberg et al. 2008), 50 powerful radio sources embedded in spiral or star-forming host galaxies. We term these objects PRONGS (powerful radio objects nested in galaxies with star formation), preliminary results of which we present here.

Whether PRONGS are an entirely new class of object is as yet unclear, but PRONGS are powerful radio objects that are physically smaller than classic FRI/FRII radio sources. Whilst Seyferts also have AGN and reside in star-forming galaxies, PRONGS typically have a higher luminosity than Seyferts. Instead, they have luminosities similar to FRI/FRII galaxies, but differ from them in being hosted by star-forming galaxies, whereas FRI/FRII host galaxies generally have SEDs characteristic of early-type galaxies. Some of the PRONGS are smaller than classical FRI/FRII galaxies, and some may be sufficiently compact to resemble CSS/GPS sources (O'Dea 1998).

We are obtaining high-resolution images of the PRONGS to resolve the radio morphology and confirm the positional accuracy so as to ensure PRONGS are not mere chance alignments. We will also determine Sersic profiles of the host galaxies to determine their true nature. Ultimately the study of PRONGS will provide another piece in the puzzle of galaxy evolution.

\section{References}

Matthews, T. A., Morgan, W. W., \& Schmidt, M., 1964, ApJ, 140, 35

Middelberg, E., et al. 2008, AJ, 135, 1276

Norris, R. P., et al. 2006, AJ, 132, 2409

O'Dea, C. P. 1998, PASP, 110, 493 